\title{
High Concentrations of Divalent Cations in Extracellular Environments Reduce in vitro Antibiotic Activity of Tigecycline
}

\author{
Jeongwoo Jo, Sunju Kim, Kwan Soo Ko* \\ Department of Microbiology, Sungkyunkwan University School of Medicine, Suwon 16419, Republic of Korea
}

\author{
Corresponding \\ Kwan Soo Ko, PhD \\ Department of Microbiology \\ Sungkyunkwan University School of \\ Medicine Suwon 16419, Republic of \\ Korea \\ Phone : +82-31-299-6223 \\ Fax : +82-31-299-6229 \\ E-mail : ksko@skku.edu
}

Received : May 12, 2021

Revised : June 14, 2021

Accepted : June 15, 2021

No potential conflict of interest relevant to this article was reported.

Copyright (C) 2021 Journal of Bacteriology and Virology

(C) This is an Open Access article distributed under the terms of the Creative Commons Attribution Non-Commercial License

(http://creativecommons.org/

license/by-nc/3.0/).
Tigecycline is known to form a complex with divalent cations such as $\mathrm{Mg}^{2+}$ and $\mathrm{Ca}^{2+}$, which is essential for a mode of action of tigecycline. In this study, we investigated the effects of levels of divalent cations on the in vitro antibacterial activity of tigecycline against two strains each of Klebsiella pneumoniae, Escherichia coli, Pseudomonas aeruginosa, and Acinetobacter baumannii. When $\mathrm{Mg}^{2+}$ or $\mathrm{Ca}^{2+}$ was added to be tantamount to criteria levels of severe hypermagnesemia or hypercalcemia in Mueller-Hinton II broth, minimum inhibitory concentrations were increased 2- to 4-fold in all strains. In media containing high concentrations of $\mathrm{Mg}^{2+}$ or $\mathrm{Ca}^{2+}$, bacterial survival rates increased significantly after 24-hour exposure to tigecycline for all strains except one $K$. pneumoniae strain. Thus, levels of divalent cations in extracellular condition might affect tigecycline activity. While testing on humans is still required, our results suggest that caution should be exercised when using tigecycline to treat infected patients with hypermagnesemia and hypercalcemia.

Key Words: Tigecycline, Divalent cation, Hypermagnesemia, Hypercalcemia

\section{INTRODUCTION}

The emergence of bacterial pathogens that are resistant to commonly used antimicrobials is one of the most serious threats to public health (1). For instance, carbapenem resistance in gram-negative bacteria raises therapeutic challenges because carbapenems have been considered the most effective antibiotics against infections caused by multi-drug resistant (MDR) or extensively drug-resistant (XDR) pathogens (2). To combat with these MDR or XDR pathogens, tigecycline is used as a last-line antibiotic (3). Bacterial isolates resistant to tigecycline have become more abundant in recent decades.

Understanding the mechanism of antibiotic action as well as the mechanism of antibiotic resistance is critical for optimizing the clinical efficacy of antibiotics while preventing the development of antibiotic resistance. Thus, it is important to study the effect of altering clinical conditions on antibiotic activity (4). Tigecycline binds to the bacterial 305 ribosome and blocks the entry of tRNA, preventing protein synthesis (5). It is known that tigecycline forms reversible complexes with divalent cations, especially $\mathrm{Mg}^{2+}$ (6). Complexation with divalent cations is important for the biological action of tigecycline. 
In this study, we investigated the effects of divalent cations in an extracellular environment on the in vitro activity of tigecycline against four species of gram-negative bacteria. The results showed that divalent cations in the extracellular environment reduced the antibacterial efficacy of tigecycline in all bacterial species investigated.

\section{MATERIALS AND METHODS}

We investigated four gram-negative bacterial species: Klebsiella pneumoniae (K. pneumoniae), Escherichia coli (E. coli), Pseudomonas aeruginosa (P. aeruginosa), and Acinetobacter baumannii (A. baumannii). For each bacterial species, two strains were examined (Table 1). In vitro antimicrobial susceptibility testing was performed on strains to determine minimum inhibitory concentrations (MICs) by the broth microdilution method, according to the Clinical and Laboratory Standards Institute guidelines (7). Tigecycline was obtained from Pfizer (Korea), and tetracycline was purchased from Sigma-Aldrich (St. Louis, MO, USA). E. coli ATCC25922 and P. aeruginosa ATCC27853 were used as control strains. MICs were determined using cation-adjusted Mueller-Hinton broth (MHBII) and MHBII with added $\mathrm{Mg}^{2+}$ or $\mathrm{Ca}^{2+}$. Magnesium sulfate heptahydrate $(24.65 \mathrm{~g})$ was dissolved in autoclaved distilled water $(87 \mathrm{~mL})$ to prepare $100 \mathrm{~mL}$ of $1 \mathrm{M}$ magnesium sulfate stock solution. Calcium chloride dihydrate $(14.70 \mathrm{~g})$ was dissolved in autoclaved distilled water $(94.5 \mathrm{~mL})$ to prepare 100

Table 1. Minimum inhibitory concentrations of each strain of four gram-negative bacteria in MHB II and MHB II with added divalent cations

\begin{tabular}{|c|c|c|c|c|}
\hline \multirow{2}{*}{ Species } & \multirow{2}{*}{ Strain no. } & \multirow{2}{*}{ Media } & \multicolumn{2}{|c|}{ MIC (mg/L) } \\
\hline & & & Tigecycline & Tetracycline \\
\hline \multirow{6}{*}{ K. pneumoniae } & \multirow{3}{*}{ ATCC 10031} & Only MHB II & 0.25 & 0.25 \\
\hline & & $+\mathrm{Mg}^{2+}$ & 0.5 & 0.5 \\
\hline & & $+\mathrm{Ca}^{2+}$ & 0.5 & 0.5 \\
\hline & \multirow{3}{*}{ B0608-134 } & Only MHB II & 0.5 & 2 \\
\hline & & $+\mathrm{Mg}^{2+}$ & 1 & 4 \\
\hline & & $+\mathrm{Ca}^{2+}$ & 1 & 4 \\
\hline \multirow{6}{*}{ E. coli } & \multirow{3}{*}{ ATCC 25922} & Only MHB II & 0.25 & 1 \\
\hline & & $+\mathrm{Mg}^{2+}$ & 0.5 & 2 \\
\hline & & $+\mathrm{Ca}^{2+}$ & 0.5 & 2 \\
\hline & \multirow{3}{*}{ B0707-025 } & Only MHB ॥ & 0.5 & $>64$ \\
\hline & & $+\mathrm{Mg}^{2+}$ & 1 & $>64$ \\
\hline & & $+\mathrm{Ca}^{2+}$ & 1 & $>64$ \\
\hline \multirow{6}{*}{ P. aeruginosa } & \multirow{3}{*}{ ATCC 27853} & Only MHB II & 8 & 16 \\
\hline & & $+\mathrm{Mg}^{2+}$ & 32 & 32 \\
\hline & & $+\mathrm{Ca}^{2+}$ & 16 & 32 \\
\hline & \multirow{3}{*}{ 08-B099 } & Only MHB II & 0.5 & 1 \\
\hline & & $+\mathrm{Mg}^{2+}$ & 2 & 4 \\
\hline & & $+\mathrm{Ca}^{2+}$ & 1 & 2 \\
\hline \multirow{6}{*}{ A. baumannii } & \multirow{3}{*}{ ATCC 19606} & Only MHB II & 1 & 1 \\
\hline & & $+\mathrm{Mg}^{2+}$ & 4 & 4 \\
\hline & & $+\mathrm{Ca}^{2+}$ & 4 & 2 \\
\hline & \multirow{3}{*}{ 06AC-02 } & Only MHB II & 0.5 & 0.5 \\
\hline & & $+\mathrm{Mg}^{2+}$ & 1 & 2 \\
\hline & & $+\mathrm{Ca}^{2+}$ & 1 & 1 \\
\hline
\end{tabular}


$\mathrm{mL}$ of $1 \mathrm{M}$ calcium chloride stock solution. Final concentrations of $\mathrm{Mg}^{2+}$ or $\mathrm{Ca}^{2+}$ in MHBll were adjusted to $2.9 \mathrm{mM}$ or 3.5 $\mathrm{mM}$, respectively, based on severe hypermagnesemia and hypercalcemia criteria levels $(3,5,8-10)$.

Additionally, we examined the survivability of the bacterial strains by measuring bacterial growth after exposure to tigecycline. Bacterial strains were cultured in MHBII at $37^{\circ} \mathrm{C}$ overnight. Cultures were diluted (1/100) in MHBII alone and MHBII with added $\mathrm{Mg}^{2+}$ or $\mathrm{Ca}^{2+}$. The final concentrations of divalent ions in $\mathrm{MHBll}$ with added $\mathrm{Mg}^{2+}$ or $\mathrm{Ca}^{2+}$ were $2.9 \mathrm{mM}$ and $3.5 \mathrm{mM}$, respectively. Tigecycline was added to the three media at the MICs for each strain. After incubation at $37^{\circ} \mathrm{C}$ for 24 hours, $\mathrm{OD}_{600}$ was measured using GeneQuant 1300 (Biochrom Ltd., Cambourne, UK); subsequently, bacterial survivability was compared among the three media. Independent experiments were performed in triplicate. To compare the two groups, Pearson $x^{2}$ tests and Fisher's exact tests were used for categorical variables and Student's $t$-test and MannWhitney $\mathrm{U}$ tests were utilized for continuous variables, as appropriate. All statistical tests were two-tailed.

\section{RESULTS AND DISCUSSION}

All strains of three gram-negative bacterial species (K. pneumoniae, E. coli, and $A$. baumannii) were susceptible to tigecycline in MHB II media alone (MICs: 0.25 to $1 \mathrm{mg} / \mathrm{L}$; Table 1). It is known that $P$. aeruginosa is intrinsically tigecycline-resistant (9). While the reference strain of $P$. aeruginosa (ATCC27853) was resistant to tigecycline, the $P$. aeruginosa clinical strain 08-B099 was susceptible. When $\mathrm{Mg}^{2+}$ or $\mathrm{Ca}^{2+}$ was added to the MHBIl media, MICs increased 2- to 4-fold in all strains, including the tigecycline-resistant $P$. aeruginosa strain ATCC27853 (Table 1).

We compared the effect of $\mathrm{Mg}^{2+}$ or $\mathrm{Ca}^{2+}$ addition on the MIC using tetracycline, an antibiotic with a mechanism of action similar to that of tigecycline (Table 1). Tetracycline MICs increased in the MHBII media with added $\mathrm{Mg}^{2+}$ or $\mathrm{Ca}^{2+}$ for all strains. Survival rates were evaluated after exposure of bacterial strains to tigecycline for 24 hours. Bacterial strains were almost completely killed in the MHBII media with no additional cations by tigecycline at the MICs (Fig. 1). However, all strains, except $K$. pneumoniae clinical strain B0608-134, showed significantly higher survivability rates in the MHBII media with additional $\mathrm{Mg}^{2+}$ or $\mathrm{Ca}^{2+}(P<0.0001)$. While K. pneumoniae B0608-134 showed high survivability against tigecycline in MHBII with additional $\mathrm{Mg}^{2+}$, survivability did not increase when $\mathrm{Ca}^{2+}$ was added to the MHBII media.

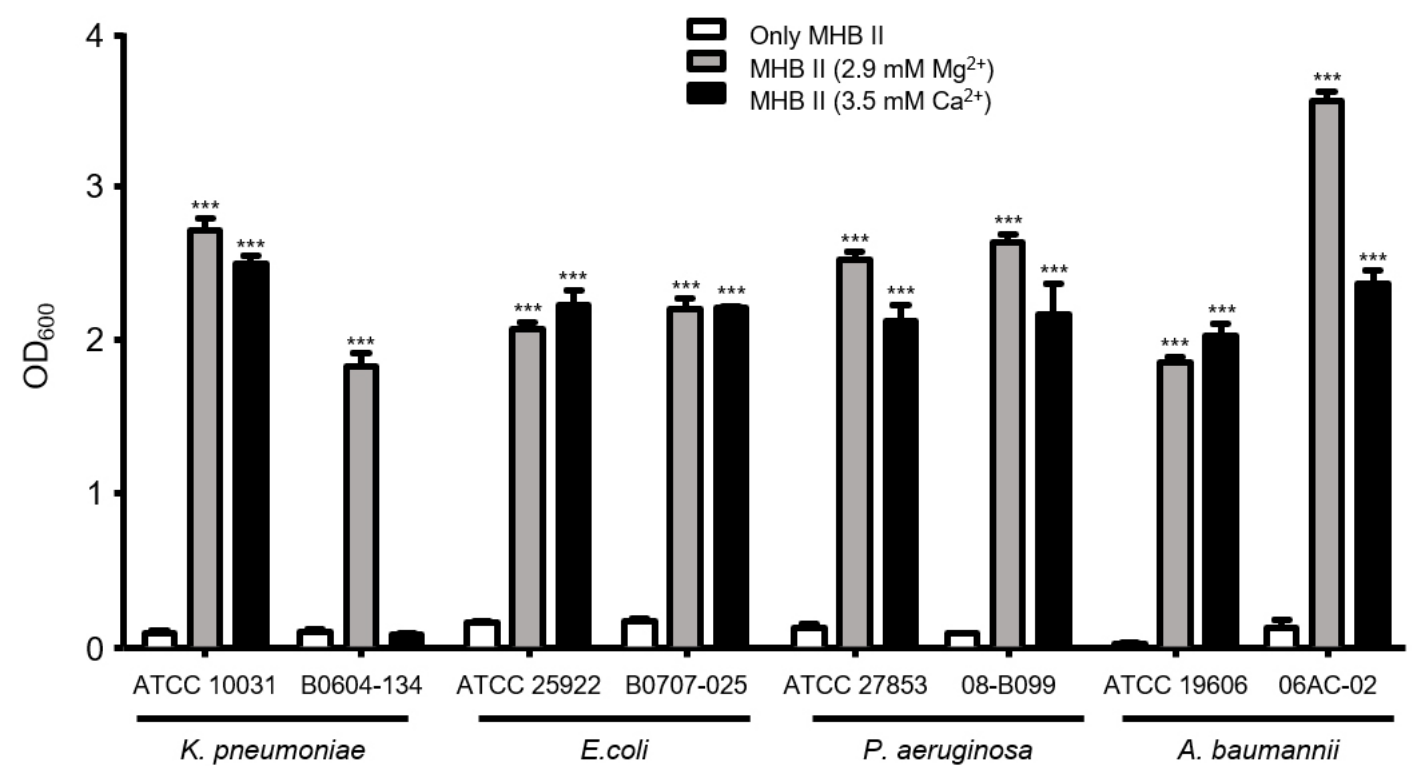

Fig. 1. Comparison of antibiotic activity of tigecycline leading to divalent cations. Each bacteria strain was cultured in MHB II with or without divalent cations for 24 hours. 
Complexation between tigecycline and divalent cations is necessary for the drug to cross the bacterial outer membrane through hydrophilic porins, such as OmpF and OmpC (11). Positively charged complexes transverse the porin following the Donnan potential caused by the distribution of ions across the outer membrane (12). In the periplasm, tigecycline dissociates from the divalent cation and becomes a protonated uncharged form, which then diffuses through the bacterial inner membrane into the bacterial cytoplasm. Thus, divalent ions are necessary for the antibiotic activity of tigecycline.

However, our data show that the concentration of divalent cations, such as $\mathrm{Mg}^{2+}$ and $\mathrm{Ca}^{2+}$, in the media influence the efficacy of tigecycline against gram-negative pathogens. In fact, high concentrations of these cations may inhibit the antibiotic activity of tigecycline. In our study, only high $\mathrm{Ca}^{2+}$ concentrations resulted in survival differences between the reference and clinical strains of $K$. pneumoniae. We tested additional clinical strains of $K$. pneumoniae and found similar results. Thus, the effect of $\mathrm{Ca}^{2+}$ on antibiotic efficacy may vary among bacterial species or strains.

Previous studies have shown that the type of medium can influence susceptibility of Enterobacteriaceae to tigecycline and tetracycline $(8-10,12-14)$. In addition, it was shown that the concentrations of $\mathrm{Mg}^{2+}$ and $\mathrm{Ca}^{2+}$ varied in the agar media used for the susceptibility tests (4). The results of these studies suggest that determination of tigecycline susceptibility should be performed with caution; moreover, fresh media should always be used during testing.

Similarly with our results, it was reported that a metal ion chelator such as EDTA could enhance the antibacterial effect of tigecycline, which was reversed by addition of divalent metal ions (15). However, in this study, we focused on the effect of levels of metal ions tantamount to criteria levels of hypermagnesemia and hypercalcemia in humans. In this regard, our study has difference with previous study. Moreover, we showed that metal ions could affect the tigecycline activity in $A$. baumannii and levels of calcium had less effect in K. pneumoniae.

The low activity of tigecycline in high divalent cation concentrations may have important implications with regard to the treatment of bacterial infections. Physiological levels of $\mathrm{Mg}^{2+}$ are critical for membrane and ribosome stabilization, neutralization of nucleic acids, and many enzymatic reactions (16). $\mathrm{Ca}^{2+}$ is also critical for maintaining physiological activity (17). As the renal system plays an important role in maintaining normal serum $\mathrm{Mg}^{2+}$ levels within a narrow range, kidney failure or end-stage renal disease causes hypermagnesemia due to a dysfunction in the process that maintains serum $\mathrm{Mg}^{2+}$ levels; it is defined as serum $\mathrm{Mg}^{2+}$ levels $>1.1 \mathrm{mmol} / \mathrm{L}$. The hypermagnesemia, which is often occurred in patients with acute bacterial infections such as bronchopneumonia and urinary tract infections, can lead to cardiovascular complications and neurological disorder (18). Our results suggest that tigecycline may be less effective in patients with high concentration of $\mathrm{Mg}^{2+}$ or $\mathrm{Ca}^{2+}$, resulted from urinary tract infections (18), regardless of the results of standard antibiotic susceptibility tests.

It is not clear why high concentrations of $\mathrm{Mg}^{2+}$ or $\mathrm{Ca}^{2+}$ lower the antibiotic efficacy of tigecycline. High levels of divalent cations forming complexes with tigecycline may delay antibiotic uptake into the bacterial cytosol. Otherwise, divalent cations may influence the function of tigecycline, inhibiting the ribosomal protein-synthesizing system. It is important to investigate why the concentrations of divalent cations lower the antibacterial activity of tigecycline. In addition, it is important that this is further evaluated in clinical studies.

In this study, we examined the effect of divalent cations on the in vitro activity of tigecycline against four species of gram-negative bacteria. Bacterial survivability increased significantly in media with high concentrations of $\mathrm{Mg}^{2+}$ or $\mathrm{Ca}^{2+}$. Our results suggest that caution should be exercised when using tigecycline to treat infected patients with high concentrations of $\mathrm{Mg}^{2+}$ or $\mathrm{Ca}^{2+}$.

\section{ACKNOWLEDGEMENTS}

This research was supported by the Basic Science Research Program through the National Research Foundation of Korea (NRF) (grant 2019R1A2C2004879). 


\section{REFERENCES}

1) Willyard C. The drug-resistant bacteria that pose the greatest health threats. Nature 2017:543:15.

2) Doi Y. Treatment Options for Carbapenem-resistant Gram-negative Bacterial Infections. Clin Infect Dis 2019;69:S565-75.

3) Stein GE, Craig WA. Tigecycline: A Critical Analysis. Clin Infect Dis 2006;43:518-24.

4) D'Amato R, Thornsberry C. Calcium and magnesium in Mueller-Hinton agar and their influence on disk diffusion susceptibility results. Curr Microbio/1979;2:135-8.

5) Sun Y, Cai Y, Liu X, Bai N, Liang B, Wang R. The emergence of clinical resistance to tigecycline. Int $J$ Antimicrob Agents 2013:41:110-6.

6) Wessels JM, Ford WE, Szymczak W, Schneider S. The Complexation of Tetracycline and Anhydrotetracycline with Mg2+ and Ca2+: A Spectroscopic Study. J Phys Chem B 1998;102:9323-31.

7) Clinical and Laboratory Standards Institute (CLSI). Performance standards for antimicrobial susceptibility testing: 29th informational supplement, M100-S29. Wayne, PA: CLSI, 2019.

8) Navarro-González J, Mora-Fernández C, García-Pérez J. Clinical Implications of Disordered Magnesium Homeostasis in Chronic Renal Failure and Dialysis. Semin Dia/2009;22:37-44.

9) Pankey GA. Tigecycline. J Antimicrob Chemother 2005;56:470-80.

10) Stewart AF. Clinical practice. Hypercalcemia Associated with Cancer. N Eng/ J Med 2005;352:373-9.

11) Chopra I, Roberts M. Tetracycline Antibiotics: Mode of Action, Applications, Molecular Biology, and Epidemiology of Bacterial Resistance. Microbiol Mol Biol Rev 2001:65:232-60.

12) Thanassi $D G$, Suh GS, Nikaido $H$. Role of outer membrane barrier in efflux-mediated tetracycline resistance of Escherichia coli. J Bacterio/ 1995:177:998-1007.

13) Hope R, Warner M, Mushtaq S, Ward ME, Parsons T, Livermore DM. Effect of medium type, age and aeration on the MICs of tigecycline and classical tetracyclines. J Antimicrob Chemother 2005;56:1042-6.

14) Torrico M, González N, Giménez MJ, Alou L, Sevillano D, Navarro D, et al. Influence of Media and Testing Methodology on Susceptibility to Tigecycline of Enterobacteriaceae with Reported High Tigecycline MIC. I Clin Microbio/ 2010;48:2243-6.

15) Deitchman AN, Singh RSP, Rand KH, Derendorf $H$. Enhanced in vitro activity of tigecycline in the presence of chelating agents. Int J Antimicrob Agents 2018:51:799-802.

16) Groisman EA, Hollands K, Kriner MA, Lee EJ, Park SY, Pontes MH. Bacterial Mg ${ }^{2+}$ Homeostasis, Transport, and Virulence. Annu Rev Genet 2013:47:625-46.

17) Dominguez DC. Calcium signalling in bacteria. Mol Microbio/2004;54:291-7.

18) Foxman B. The epidemiology of urinary tract infection. Nat Rev Uro/2010;7:653-60. 